\title{
The status of rabies in Ethiopia: A retrospective record review
}

\author{
Asefa Deressa ${ }^{1}$, Abraham $\mathrm{Ali}^{1}{ }^{1}$, Mekoro Beyene ${ }^{1}$, Bethelehem Newaye Selassie ${ }^{1}$, Eshetu Yimer $^{2}$, Kedir Hussen $^{1}$
}

\begin{abstract}
Background: Rabies, a viral disease that affects all warm-blooded animals, is widespread in many regions of the world. Human rabies, transmitted by dogs is an important public health issue in Ethiopia. To-date, effective rabies control program still remains to be a reality and needs to be strengthened..

Objective: Reviewing of recorded data to generate information on the status of rabies in Ethiopia for the year 20012009.

Methods: A retrospective data were used from the Ethiopian Health and Nutrition Research Institute rabies case record book registered between 2001 and 2009.

Results: The fatal human cases during the period of study were 386 humans with annual range of 35 to 58 . The over all post exposure treatment for humans was 17,204 within and around Addis Ababa. During the same period, 20,414 suspected rabid animals were clinically examined; nevertheless, only $10 \%$ were positive for rabies. Among 3,460 animal brains investigated in the laboratory with FAT, $75 \%$ were confirmed as rabies positive. The production and distribution of anti-rabies vaccine reached 130,673 treatment doses for human vaccine and 85,055 doses for animal vaccine respectively in the period of 2001-2009.

Conclusion: The recorded data showed the underestimate of rabies diagnosis, post exposure prophylaxis and fatal human cases, which could be attributed due to the absence of national rabies surveillance system. Therefore, It is of paramount importance to assess and map the national picture of rabies within a given time interval to launch a national rabies control strategy. [Ethiop. J. Health Dev. 2010;24(2):127-132]
\end{abstract}

\section{Introduction}

Rabies is a deadly disease caused by a virus. It is the most serious zoonotic disease that some one might encounter. The rabies virus is released in the saliva of an infected animal. The animal usually contracts rabies from the bite of an infected animal. The virus may also enter the body if the mucous membranes (the wet part of the eyes, nose, or mouth) or a scratch or break in the skin have contact with saliva containing the rabies virus. Once the rabies virus enters the body, it begins to multiply in the area near the entry site. If the infection is not stopped at this point, the virus will eventually invade the nerve cells in the area. Once the virus is in nerve tissue, it travels along the nerve to the center of multiplication (the brain). The virus may then spread to the salivary glands or other parts of the body. This incubation period lasts a varying amount of time; it can range from days to years, but the average length is 3-8 weeks. Though, rabies is one of the vaccine preventable diseases, it is practically difficult and irreversible for medical treatment after the on-set of clinical manifestation of the disease. In spite of the availability of safe and effective preventive tools in global market, the burden of the disease could not be reduced in developing countries.

In 1998 , more than 33,000 people died world wide because of rabies (1). Most of those deaths occurred in tropical developing countries (2). Canine rabies and hence human exposure can be controlled by intervening in the animal reservoir (3). The domestic dog is the most important vector of human exposure (4). Annual reports of the Ethiopian Health and Nutrition Research Institute indicated a total of 488 human deaths that occurred in 1964 and 1975 (5).

Empirical observation and models of the transmission of canine rabies indicate that rabies can be eradicated if $70 \%$ of the dog population is vaccinated repeatedly to achieve herd immunity $(6,7)$.

Stray dogs that are not accessible to mass vaccination can reduce the coverage achievement. Oral vaccines that could reach ownerless dogs are not yet on the market (8). Non-selective elimination of stray dogs to reduce the reservoir population is no longer recommended as a strategy against rabies by WHO (9), since it increases population turnover and decreases herd immunity (10), while public opposition to dog removal can lead to the failure of rabies control programs (11). In Ethiopia domestic dogs are the principal reservoir of rabies (12). Studies on dog ownership pattern and awareness of rabies in Addis Ababa showed that $90.7 \%$ of the dog owners manage dogs for the safeguarding of their properties from theft out of which $52 \%$ of them are with out regular vaccination (13). World human mortality from endemic canine rabies was estimated to be 55000 deaths per year (90\% CI: $24500-90800)$ with $56 \%$ of the deaths estimated to occur in Asia and 44\% in Africa (14).

${ }^{1}$ Ethiopian Health and Nutrition Research Institute, P.O.B.1242. Addis Ababa, Tele: $+2512751522 / 753470$;

${ }^{2}$ AU/IBAR - African Union/Inter-African Bureau for Animal Resources, Nairobi. 
In Ethiopia rabies is an important disease that has been recognized for many centuries. According to Richard Pankhurst, the first and only recorded of rabies case in Addis Ababa occurred in August 1903 (15). The incidence of human post exposure treatments and human rabies cases per million population of Ethiopia were 73.6 and 12.6 , respectively (16). It is one of the public health concerns which need formulation of intervention strategy. In Africa, the highest recorded human death due to rabies for the year 1998 was 43 and reported from Ethiopia.

The annual mass vaccination coverage is limited due to various factors. According to the annual report of Ethiopian Health and Nutrition Research Institute in the year 1997, the average anti-rabies vaccine production for animal use was about 8000 doses per year which was below the demand for domestic consumption.

This study also revealed that there was high occurrence of rabies in and around Addis Ababa due to the poor management of owned dogs and the presence of high population of unvaccinated stray dogs. Similarly, in most hard to reach areas of Ethiopia dogs are kept for hunting and protection of their owner's property. One can estimate that there might be one owned dog per five house-holds at national level, but epidemiological data on dog demography is not yet established at national level. However, Addis Ababa's dog population is estimated to be $150,000-200,000$ of which $50 \%$ may be household dogs and the rest are stray dogs (17). Regarding the dog management in Ethiopia, it is difficult to identify an overlap between the household and stray dogs with no clear-cut distinction between the two. Thus, except in few cases one cannot estimate with certainty between household and stray dogs in selected parts of Ethiopia. Moreover, it is difficult to estimate the prevalence and incidence of rabies at national level due to the absence of laboratory diagnosis and recorded data at different health facilities of the Country. With this background, the purpose of this study was to review and examine the status of rabies in Ethiopia from recorded data between 2001 and 2009 at the Ethiopian Health and Nutrition Research Institute.

\section{Methods}

A retrospective record review for the period of 2001 and 2009 was carried out at EHNRI using the rabies record book. In this record book, residents from the Metropolitan, Addis Ababa and out side of Addis Ababa within $40 \mathrm{~km}$ radius and victims of animal bite were the sources of the sampling population. The sample size was based on the incidence of animal bite and purposive sampling of retrospective record. For this case record book was designed to diagnose: 1) humans who seek for post exposure treatment after the bite of rabies suspected and /or rabies infected animals and saliva contacts 2) suspected animals if accessed alive, killed or dead. According to the World Health Organization (WHO) for the year 1993 the post exposure prophylaxis algorithm was used for the screening of rabies exposed humans and animals. Suspected humans and animals who visit Ethiopian Health and Nutrition Research Institute were registered by address, age, sex, site of bite, date of bite, type of bite, the status of biting animal (whether it has disappeared or killed, vaccinated or non-vaccinated), clinical manifestations like single or multiple bite, hydrophobia for both human and animal and aerophobia for human were among the parameters used for clinical observation. Humans who were supposed to take the full course of anti-rabies treatment were directed to Health centers and hospitals for vaccination. According to the WHO standard, animal bite victims were supposed to be vaccinated 0.5 c.c. on days zero, seven, twenty eight or twenty one $(0,7,28$ or 21$)$ as pre-exposure prophylaxis, where as rabid animal and human contact and/or bite victim individuals took the full course of treatment on days zero, three, seven, fourteen and twenty eight $(0$, $3,7,14$ and 28) as post exposure prophylaxis. In cases of Fermi type anti-rabies vaccine produced at EHNRI a dose of 5 c.c. subcutaneous injections around the umbilicus for fourteen consecutive days and a booster dose of 5 c.c. within ten days interval was used as a prescription. Suspected animals were kept in standard animal cage for clinical observation and quarantined for 10 consecutive days at the Ethiopian Health and Nutrition Research Institute. Brains from all dead animals including humans during post mortem examination were aseptically collected and brain smears are prepared after which it was subjected to Fluorescent Anti-body Test (FAT) which is a gold standard for rabies diagnosis. Unknown field samples were diagnosed using standard operating procedures of FAT and positive control. The mouse inoculation test was also used in cases of in-vivo trail and further laboratory confirmation. The fatal human rabies cases were obtained from the records of human fatal case record book in which date of bite, date of illness, length of illness, age, sex, and date of death were registered. A descriptive statistics was performed to generate frequencies and percentages.

\section{Results}

\section{Rabies Diagnosis and post exposure treatment}

Rabies diagnosis was performed on brain samples that are submitted to the EHNRI laboratory as suspected of being affected by rabies. The brain sample was either from live animals' or killed (dead) animals' brain. Between 2001 and 2009, a total of 20,414 animals that bite humans were brought to the Institute for clinical examination. After clinical observation and 10 days quarantine period $18,243(89 \%)$ of the animals were certified as free from rabies for that specific observation period and vaccination of animals was advised to maintain the observed health status of the animals (Table 1). Out of 3,460 brain samples of different animals, mainly dogs, submitted for examination, 2,593 (75\%) were found to be positive for rabies by the FAT (Table 2). The proportion of rabies among dogs was $88.7 \%$ in 2004 , 
where as it was $68.65 \%$ in 2008 (Table 1). Some humans that were bitten by known rabid animals (confirmed positive) and those bitten by animals that bit and disappeared or bitten by unknown animals were given post exposure anti-rabies prophylaxis. Between 2001 and 2009, a total of 11,017 (64\%) humans from Addis Ababa and 6,187 (35.96\%) humans from areas outside of Addis Ababa were advised to take post exposure anti- rabies prophylaxis (Table 3). Annual post exposure prophylaxis for human rabies ranges between 1026 and 1580 every year for the last nine years in Addis Ababa. It ranged from 300 to 1922 for areas outside Addis Ababa.

Table 1: Clinical observation and quarantine of animals suspected of rabies from Addis Ababa and surrounding areas between 2001 and 2009.

\begin{tabular}{lccc}
\hline \multicolumn{1}{c}{ Species } & $\begin{array}{c}\text { Total \# under clinical } \\
\text { observation \& } \\
\text { quarantine }\end{array}$ & $\begin{array}{c}\text { Animals free from rabies } \\
\text { after 10 days of quarantine } \\
\text { No. (\%) }\end{array}$ & $\begin{array}{c}\text { \% positive for } \\
\text { rabies } \\
\text { No. (\%) }\end{array}$ \\
\hline Dog & 20113 & $18102(90 \%)$ & $2011(10 \%)$ \\
Cat & 265 & $125(47 \%)$ & $140(53 \%)$ \\
Others (donkey, monkey, horse, cattle) & 36 & $16(44 \%)$ & $20(55 \%)$ \\
\hline Total & 20414 & $18243(89 \%)$ & $2171(10.63 \%)$ \\
\hline
\end{tabular}

Table 2: The Brain of animals examined for rabies from Addis Ababa and surrounding areas between 2001 and 2009.

\begin{tabular}{lllllll}
\hline \multirow{2}{*}{ Year } & \multicolumn{2}{c}{ Dog } & \multicolumn{2}{c}{ Cat } & \multicolumn{2}{c}{ Others } \\
\cline { 2 - 6 } & $\begin{array}{l}\text { Brain } \\
\text { examined }\end{array}$ & $\mathbf{N}$ No (\%) positive & $\begin{array}{l}\text { Brain } \\
\text { examined }\end{array}$ & No (\%) positive & $\begin{array}{l}\text { Brain } \\
\text { examined }\end{array}$ & No (\%) positive \\
\hline 2001 & 379 & $274(72)$ & 15 & $11(73)$ & 1 & 0 \\
2002 & 1259 & $919(73)$ & 20 & $17(85)$ & 19 & $14(73.68)$ \\
2003 & 400 & $302(75)$ & 12 & $10(83)$ & 10 & $5(50)$ \\
2004 & 115 & $102(88.7)$ & 9 & $5(55)$ & 5 & $3(30)$ \\
2005 & 297 & $237(79.8)$ & 17 & $12(70)$ & 3 & $2(66)$ \\
2006 & 280 & $193(68.9)$ & 11 & $5(45)$ & 7 & $571.43)$ \\
2007 & 240 & $202(84)$ & 15 & $10(66)$ & 7 & $3(42.8)$ \\
2008 & 67 & $46(68.65)$ & 12 & $10(83)$ & 10 & $7(42.8)$ \\
2009 & 224 & $183(81.7)$ & 15 & $11(73)$ & 11 & $5(45)$ \\
Total & 3261 & $2458(75.37)$ & 126 & $91(72)$ & 73 & $44(60)$ \\
\hline
\end{tabular}

Table 3: Post Exposure Prophylaxis (PEP) against rabies for those from Addis Ababa and outside between 2001 and 2009

\begin{tabular}{lllllllllll}
\hline Location/Year & 2001 & 2002 & 2003 & 2004 & 2005 & 2006 & 2007 & 2008 & 2009 & Total \\
\hline In A.A*. & 1240 & 1244 & 1522 & 1580 & 1081 & 1050 & 1130 & 1026 & 1144 & $11017(64.4 \%)$ \\
Out A.A. & 300 & 1922 & 520 & 770 & 567 & 477 & 522 & 513 & 596 & $6187(35.96 \%)$ \\
Total & 1540 & 3166 & 2042 & 2350 & 1648 & 1527 & 1652 & 1539 & 1740 & 17204
\end{tabular}

* In side Addis Ababa and Out side of Addis Ababa within $40 \mathrm{~km}$ radius

Anti-rabies vaccine production for humans and animals:

The Fermi type adult sheep brain nervous tissue vaccine is produced at the Ethiopian Health and Nutrition Research Institute (EHNRI). The vaccine is produced both for humans and animals use. It is distributed on demand bases to various regions of Ethiopia.

Human anti- rabies vaccine production:

The vaccine is a 5\% suspension of phenolized sheep brain tissue. In the last nine years a minimum of 6,263 and a maximum of 21,832 doses of the human rabies vaccine were produced and distributed every year (Table $6)$.
Animal anti-rabies vaccine:

It is a $20 \%$ phenolized sheep brain nervous tissue vaccine. The annual production of animal rabies vaccine was a minimum of 4363 and a maximum of 21400 prophylactic doses produced and dispatched for the last nine years (Table 6). The over all production and distribution of 130,673 doses of human and 85,055 doses of animal vaccine during the period of 2001-2009 to the various regions is an indicator of the efforts to met the demand for vaccines against rabies in the country (Table 6). The human rabies vaccine doses distributed to regions during $2004-2009$ showed that out of 102,098 doses of vaccine, the highest dose of $58,618(57 \%)$ was dispatched to the Oromiya Regional State and the lowest dose 19 (0.02\%) went to the Afar Regional State. 
Fatal human rabies cases

The fatal human cases in 2001-2009 were 386 humans with annual range of 35 to 58 (Table 4). The age and sex specific distribution showed that the most fatal cases were $42 \%$ from the age group 0-14 category (Table 5). According to this record $66.66 \%$ of deaths were males and $33.33 \%$ were females.
Clinical observation of cats and other animals suspected of rabies:

Clinical observation of cats suspected of rabies indicated out of 265 clinical observations $53 \%$ were positive for rabies and $47 \%$ were certified as provisionally free from rabies after 10 days quarantine period. Among 36 other domestic and wild animals like donkey, cattle, horse, fox and monkey suspected of rabies, $20 \%$ were confirmed positive for rabies.

Table 4: Sex and age specific fatal human rabies cases from Addis Ababa and surrounding areas between 2001 and 2009.

\begin{tabular}{|c|c|c|c|c|c|c|}
\hline \multirow[t]{2}{*}{ Age Group } & \multicolumn{2}{|c|}{ Male } & \multicolumn{2}{|c|}{ Female } & \multicolumn{2}{|c|}{ Total } \\
\hline & \# & $\%$ & \# & $\%$ & \# & $\%$ \\
\hline $0-14$ & 110 & $66.66 \%$ & 55 & $33.33 \%$ & 165 & 42.74 \\
\hline $15-49$ & 86 & $53.42 \%$ & 75 & $46.58 \%$ & 161 & 41.71 \\
\hline $50+$ & 35 & $58.33 \%$ & 25 & $41.66 \%$ & 60 & 15.54 \\
\hline Total & 231 & 59.84 & 155 & 40.16 & 386 & 100 \\
\hline
\end{tabular}

Table 5: Human rabies vaccine doses distributed by regions between 2004 and 2009

\begin{tabular}{|c|c|c|c|c|c|c|c|}
\hline \multirow{2}{*}{$\begin{array}{l}\text { Administrative } \\
\text { Region }\end{array}$} & \multicolumn{5}{|c|}{ Year } & & \multirow[t]{2}{*}{ Total } \\
\hline & 2004 & 2005 & 2006 & 2007 & 2008 & 2009 & \\
\hline Oromiya & $6482^{*}$ & 8056 & 10847 & 13353 & 12887 & 6993 & $58,618(57 \%)$ \\
\hline Addis Ababa & 1555 & 1170 & 1428 & 2025 & 2074 & 829 & $7,281(7 \%)$ \\
\hline SNNP & 1362 & 1094 & 2820 & 2913 & 3293 & 2686 & $14,168(13.87)$ \\
\hline Tigrai & 404 & 744 & 847 & 1281 & 1303 & 1133 & $5,712(6 \%)$ \\
\hline Afar & 0 & 0 & 0 & 3 & 0 & 16 & $19(0.02 \%)$ \\
\hline Somali & 35 & 12 & 29 & 88 & 1436 & 647 & $2,247(2.2 \%)$ \\
\hline Benshangul & 119 & 138 & 207 & 341 & 306 & 118 & $1,229(1.2 \%)$ \\
\hline Gambella & 5 & 26 & 31 & 8 & 88 & 47 & $205(0.20 \%)$ \\
\hline Harari & 0 & 0 & 600 & 500 & 0 & 29 & $1,129(1.1 \%)$ \\
\hline Diredawa & 85 & 25 & 967 & 1079 & 1078 & 71 & $3,305(3.28 \%)$ \\
\hline Amhara & 339 & 335 & 967 & 3901 & 2804 & 1163 & $9,509(9.31 \%)$ \\
\hline Total & $11322^{*}$ & 11294 & 14832 & 16996 & 20334 & 21832 & \\
\hline
\end{tabular}

${ }^{*}$ One bottle of vaccine is equivalent to 100c.c. treatment dose

Table 6: Annual human and animal anti rabies vaccine production in EHNRI between 2001 and 2009.

\begin{tabular}{llllllllll}
\hline Dose/year & $\mathbf{2 0 0 1}$ & $\mathbf{2 0 0 2}$ & $\mathbf{2 0 0 3}$ & $\mathbf{2 0 0 4}$ & $\mathbf{2 0 0 5}$ & $\mathbf{2 0 0 6}$ & $\mathbf{2 0 0 7}$ & $\mathbf{2 0 0 8}$ & $\mathbf{2 0 0 9}$ \\
\hline $\begin{array}{l}\text { Animal } \\
\text { vaccine }\end{array}$ & 21400 & 4363 & 7634 & 10118 & 5000 & 11380 & 6640 & 9260 & 9260 \\
$\begin{array}{l}\text { Human } \\
\text { vaccine* }\end{array}$ & 6263 & 19800 & 8000 & 11322 & 11294 & 14832 & 16996 & 20334 & 21832 \\
\hline \begin{tabular}{l} 
Total \\
\hline
\end{tabular} & $\mathbf{2 7 6 6 3}$ & $\mathbf{2 4 1 6 3}$ & $\mathbf{1 5 6 3 4}$ & $\mathbf{2 1 4 4 0}$ & $\mathbf{1 6 2 9 4}$ & $\mathbf{2 6 2 1 2}$ & $\mathbf{2 3 6 3 6}$ & $\mathbf{2 9 5 9 4}$ & $\mathbf{3 1 0 9 2}$ \\
\hline
\end{tabular}

\section{Discussion}

The proportion of dog bite cases and fatal human cases were increasing during the observation period. This is in agreement with the previous works of Yimer and et al. (12). This indicates that in spite of the initiation of the rabies diagnosis and post exposure treatment routine service delivery, dog bite and fatal human cases were not declining.

Rabies clinical observation and quarantine of animal data revealed that $90 \%$ of clinically observed animals were free from rabies after 10 days quarantine period. Ten percent of them were positive during the observation period which was also confirmed by laboratory findings.. This clinical observation shows that all biting dogs are not infected by rabies. Some times under Ethiopian situation, people move door-to-door from one house to another for cultural holidays and other religious festivals which can trigger owned dogs to bark and bite visiting gust in its compound under normal condition. The aggressive behavioral change of male dogs during mating and breeding season can also contribute for biting due to 
physiological reaction with out rabies clinical manifestation. Similarly, female dogs that gave birth are aggressive and can bite humans under normal condition to protect the newly born puppies from some individuals who want to take and abandon the puppies immediately after birth.

Based on the 10 days clinical observation at the Ethiopian Health and Nutrition Research Institute, dogs were the first most important sources of rabies and cats are next to dogs in Ethiopia. This was evident from the 3,261 total dog brain samples collected for the last nine years out of which $2,458(75 \%)$ were positive cases. Among 121 cat brains examined 91 (72\%) were positive for the same period. The laboratory confirmed rabies cases in other wild (hyenas, foxes) and domestic (dog, cattle, donkey and horse) animals showed $60 \%$ positive cases. Hence, the interface of wild and domestic animals in grazing areas, water points and backyard waste disposal areas can facilitate the circulation of the virus in the country. This report is supported with previous records by Yimer et al. (12), Fekadu (14) and Ayalew (18) that dogs are primary cause and cats are secondary for fatal human rabies cases and responsible in maintaining as well as dissemination of rabies in Ethiopia.

The number of humans who received post exposure prevention against rabies shows a range of 150 to 300 monthly distributions of animal bite cases with constant risk of contracting the disease from the bite of the animals. Although, some dog bite out - break cases from North Wollo, East Gojam and Jijiga zones have been included in this report, still it is considered as an underestimate of the national picture as most victims of dog bite in the rural Ethiopia have access to traditional healers prior to the rabies diagnosis facility in modern health institutions. The deep rooted traditional practice of pretending to treat rabies in Ethiopia interferes with getting the real magnitude of he problem. The existing experience indicates that most human rabies victims come to EHNRI laboratory either after the loss of life from family member or after exhausting the traditional medicinal intervention. By the time the victim arrives the disease has already developed with the manifestation of clinical symptoms and cases are not in a position to be reversed by medical care. There was a remarkable difference between areas within and out side Addis Ababa to receive post exposure prophylaxis This can be justified as people from in accessible areas prefer to seek medical care from traditional healers instead of traveling long distance to Addis Ababa as there is no rabies consultation service in the nearby.

The age and sex specific distribution showed that most fatal cases were from $0-14$ age category with $67 \%$ deaths of males and $33 \%$ deaths of Females from the same age category. The minimum deaths were recorded in 50 years and above age category.
This can be explained as most of the victims are infants and school children who are closely playing with pets at home and even in streets under Ethiopian situation. In some cases children do not care for further treatment after they get wound treatment on the site of animal bite. In the contrary, some elders are well aware of the danger of rabies and look for medical care.

Most fatal human rabies cases recoded at EHNRI during the last nine years were associated with herbal remedies whereby majority of human rabies cases were helped exhaustively by traditional healers. In the mean time, most human rabies cases were manifested before they reach health facilities. In some cases victims were advised by healers and even to the extent they believe not to cross rivers and travel to health facilities in search of medical care before 40 days. According to the healers, if there is no clinical sign during this period the healers certify that the dog bite cases are free of rabies. This is scientifically not accepted due to various factors. The development and incubation of rabies virus is influenced by: 1) site of bite whether it is close or far from the center of multiplication of the virus which is the brain 2) the type of wound can also be a factor to influence the incubation period. In this regard, deep wound is likely to be with high inoculation doses of the virus in to the site of the bite. Thus, the public should be aware to go to health facilities immediately after the bite of the animal for further medical care and consultation.

The maximum and minimum production and distribution of human and animal vaccine during 2001- 2009 indicates the increasing supply of rabies vaccine both for humans and animals throughout Ethiopia. Most animal anti-rabies vaccine is dispatched to the Addis Ababa Region Agricultural Bureau. However, during the 2002 fiscal year the production has decreased due to importation of animal vaccine as alternative sources of vaccine.

The human rabies vaccine doses distributed to regions during 2004-2009 showed that out of 102,098 doses of vaccine, the highest dose 5,861 (57\%) was distributed to Oromiya Regional State and the lowest dose 19 (0.02\%) to Afar Regional State. This could be explained by the higher incidence of dog bites that increased the vaccination demand in Oromiya Regional State compared to the Afar Regional State. The number of owned dogs versus the number of stray dogs and the dog population dynamics in general could be different in both regional states. However, the distribution of a total of 130,673 doses of human and 85,055 doses of animal vaccine during the period of 2001-2009 to various regions was an indication of the magnitude of rabies both in humans and animals throughout the country.

In general, the data on fatal human rabies cases and diagnosis are an underestimate of the crude death and prevalence rates at national level sine most deaths occur

Ethiop. J. Health Dev. 2010;24(2) 
in the community far into hard to reach areas where there is no laboratory facility and proper record for screening. Thus, the current data collected at EHNRI during the observation period are compatible with the previous retrospective data compiled by Yimer et al (12), Ayalew (18) and Fekaku (14) which indicated the high proportion of fatal human rabies cases in Ethiopia.

\section{Conclusion and recommendation:}

The production size and distribution of human antirabies vaccine for domestic supply per annum has increased from six thousand to twenty thousand doses for the last nine years, however it does not meet the projected domestic demand of eighty thousand doses at national level. The retrospective data for rabies diagnosis, post exposure prophylaxis and fatal human cases were underestimated due to the absence of rabies diagnosis laboratory across the country. It is of a paramount importance to assess and map the national picture of rabies within a given time interval to launch a national rabies control strategy.

\section{Acknowledgments}

The authors would like to express their gratitude to the animal attendants Ato Daniel Legesse and Ato Afework Kabtyimer for the valuable contributions they made in providing animal management service during quarantine period.

\section{References}

1. World survey of rabies No. 34 for the year 1998. Geneva: WHO document; Available from: URL: http//whqlibdoc.who.int/hq/199/who_CDC_CSR_A PH 99.6.pdf.

2. Warrel DA, Warrel MJ. Human rabies: A continuing challenge in the tropical world. Schweizenishe Wochenschnft 1995;125:879-85.

3. Cleaveland S, Feire EM, Kaare M, Coleman PG. Estimating human rabies mortality in the United Republic of Tanzania from dog bite injuries. Bulletin of the WHO 2002;80:304-10.

4. Wandeler Al, Matter HC, Kappeler A, Budde A. The ecology of dogs and canine rabies: a selective review Soentifique et Technique Office International des Epizooties 1993;12:51-57.

5. Mebatsion T, Cox JH, Frost JW. Isolation and characterization of 115 street rabies virus isolated from Ethiopia by using monoclonal antibodies: identification of 2 isolates Mokola and Lagos bat virus. J. Infectious Diseases 1992;166:972-77.
6. WHO. Guidelines for dog rabies control. Geneva: World Health Organization; 1987.WHO document VPH/83.43:Rev.1. Available from: URL: http// whqlibdoc:who.int/hq/pre-wholis/vph_83.43_Rev. 1.pdf.

7. Coleman PG, Dye C. Immunization coverage required to prevent outbreaks of dog rabies. Vaccine 1996;14:185-6.

8. WHO. Oral immunization of dogs against rabies. Report of the South WHO Consultation, Geneva, 2425 July 1995. Geneva: World Health Organization; 1998. WHO document; Available from: URL: http//whqlibdoc:who.int/hq/prewholis/vph_83.43_Rev.1.pdf.

9. Bogel. K. Guidelines for Dog Rabies control. Geneva: WHO; June 1987.

10. Matter HC, Kharmach H, Haddad N, Ben Youssef S, C, Ben Khelifa R. Test on three bait types for oral immunizationof dogs against rabies in Tunsia. American Journal of Tropical Medicine and Hygiene 1995;52:489-95.

11. Beran GW. Urban rabies. In: Baer GM, editor. The natural history of rabies. Boca Racon (FL): CRC press; 1991: 428-43.

12. Yimer E, Neway B, Girma T, Mekonnen Y, Yoseph B, Badeg Z, Mekoro B, and Abebe B. Situation of rabies in Ethiopia: a retrospective study 1990-2000. Ethiop J Health Dev 2002;16(1):105-112.

13. EHNRI. Zoonoses and anti-rabies vaccine production annual report 1997. Ethiopian Health and Nutrition Research Institute. 1997, Addis Ababa, Ethiopia.

14. Fekadu, M. Human rabies surveillance and control in Ethiopia. In: Proceedings of the Southern and Eastern Africa Rabies Group Meeting Nairobi, Kenya 4-6 March 1997.

15. Pankhrust R. An introduction to the medical history of Ethiopia. the Red sea press. Inc. Trenton, New Jersey: 1990-93-101.

16. Bogel K, MoTschwiller E. Incidence of rabies and post exposure treatment in developing countries. Bulletin of the WHO 1986;64(6):883-887.

17. Ministry of Agriculture Veterinary Services Annual report, 2000.

18. Yilma A. Analysis of 159 human rabies cases in Ethiopia In: Kuwert E, Meirieux C, Koprowski H, Bogel H eds, Rabies in the Tropics. New York: Springer-Verlag 1985. 